\section{CME Große Portion CME-Punkte mit e.Med!}

Auch 2015 haben wir wieder ausgewählte CME-Kurse

für Ihre hausärztliche Fortbildung in der Sommerakademie

von Springer Medizin zusammengestellt.

\begin{abstract}
Nutzen Sie die Sommerzeit, um reichlich CME-Punkte zu sammeln und um sich schnell und effizient fortzubilden!

Sichern Sie sich Ihre Punkte online in der Springer Medizin Sommerakademie: springermedizin.de/sommerakademie.

Teilnahme mit e.Med oder dem Zeitschriftenabo möglich.

Mit e.Med haben Sie nicht nur Zugriff auf die aktuelle Sommerakademie, Sie können das komplette Angebot von Springer Medizin nutzen. Sie haben Zugriff auf alle Inhalte von springermedizin.de, können alle Fachzeitschriften inklusive Archive im Volltext online lesen - zusätzlich erhalten Sie eine gedruckte Fachzeitschrift Ihrer Wahl nach Hause.
\end{abstract}

Vor allem erhalten Sie Zugang zu allen CME-Fortbildungen der e.Akademie: Mit rund 1600 Fortbildungskursen, davon über 500 CME-zertifiziert, ist das Lernportal von Springer Medizin Deutschlands erste Adresse für Online-CME-Fortbildung.

Sie haben außerdem die Möglichkeit uneingeschränkt in unseren vielen englischsprachigen Journals zu recherchieren.

e.Med 30 Tage kostenlos und unverbindlich testen: www.springermedizin.de/eMed

Unser Kundenservice steht Ihnen wochentags (10-17 Uhr) kostenlos zur Verfügung: Tel. 08007780777

kundenservice@springermedizin.de

Ihre CME-Redaktion

\section{Von COPD bis}

\section{Rheuma}

In unserer Sommerakademie haben wir wieder eine breite Auswahl besonders praxisrelevanter und aktueller Themen für Sie zusammengestellt (ab S.4). Auf S. 43 erwarten Sie weitere potenzielle Punkte mit unseren interaktiven "Sahne.stücken“. Den CME-Weiterbildungsbeitrag „Therapie der Alkoholabhängigkeit" finden Sie ab S.57. Füttern Sie Ihr Punktekonto mit dieser Ausgabe! Viel Spaß beim Lesen und Fortbilden!

Melanie Goldschmidt Redakteurin

\section{"Schwierige Lebensumstände" entbinden nicht von der Fortbildungspflicht}

Das Bundessozialgericht hatte kürzlich zu entscheiden, ob der Zulassungsentzug gegen eine Ärztin gerechtfertigt war. Die Praxisinhaberin hatte ihrer KV trotz Honorarkürzung und wiederholter Mahnung keine Nachweise über die gesetzlich geforderte Fortbildung vorlegen können. Selbst die zweijährige Nachfrist ließ sie verstreichen. Sie führte „schwierige private Umstände" zur Entschuldigung an, die nicht näher konkretisiert wurden. Auch ein Wohlverhalten in dem anschließenden
Fortbildungszeitraum ändert laut BSG nichts an der Verhältnismäßigkeit des Zulassungsentzugs. Unverhältnismäßig könnte der Entzug dann sein, wenn der Nachweis nur um wenige Stunden verfehlt wird, so die Richter. Ärzten, die sich an der Erfüllung ihrer Fortbildungspflicht gehindert sehen, raten die Sozialrichter, gegebenenfalls das vollständige oder hälftige Ruhen ihrer Zulassung zu beantragen.

www.aerztezeitung.de

\title{
e.Curriculum Geriatrie: gratis, interaktiv \& 5 CME-Punkte pro Modul!
}

Erfahren Sie in dieser Fortbildung alles über die Besonderheiten bei geriatrischen Patienten - und sammeln Sie 5 CMEPunkte pro Modul! Mehr auf Seite 22.

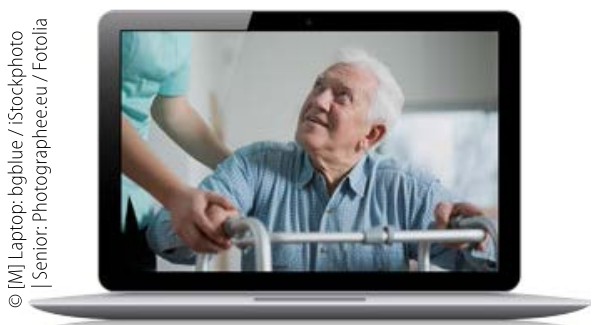

- Modul 1: Bewegung, Immobilität und Sturzgefahr

- Modul 2: Depression, Suizidalität und Demenz

Modul 3: Notfallmedizin

Modul 4: Polymedikation

Modul 5: Ernährung

Kurse online: www.springermedizin.de/ ecurriculum-geriatrie

In Kooperation mit: Robert Bosch Stiftung, DGIM, DGG

\section{CME-Punkte für Alkoholtherapie}

Kennen Sie die Häufigkeit von Alkoholkonsum und -abhängigkeit in Deutschland? 2012 ging man in Deutschland von einem Pro-Kopf-Konsum von 9,5 1 reinem Alkohol und von 74.000 alkoholbedingten Todesfällen aus. Die Zahl der Alkoholabhängigen wird auf bis zu 1,6 Mio. geschätzt. Dazu kommen ca. 2 Mio. Menschen mit Alkoholmissbrauch und ca. 5 Mio. mit riskantem Konsum.

Können Sie eine Abhängigkeit diagnostizieren und verstehen Sie die therapeutischen Prinzipien? Für die Therapie alkoholbezogener Störungen liegen zahlreiche Studien und Therapieempfehlungen vor. Lernen Sie sinnvolle psychotherapeutische Interventionen kennen und welche Medikamente zur Rückfallprophylaxe sinnvoll sind - damit können Sie Ihren Patienten helfen, einen für sie gangbaren Weg aus der Suchtfalle zu finden.

Prüfen Sie Ihr Wissen, beantworten Sie die zehn Fragen online und kassieren Sie drei CME-Punkte. Den Kurs finden Sie im Heft ab Seite 57.

Viele Deutsche sind an die Flasche gefesselt Sprengen Sie die Ketten!

\section{(red)} d)

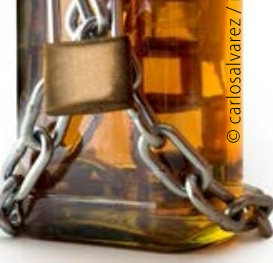

